\title{
IgA Nephropathy, Liver Disease-associated
}

National Cancer Institute

\section{Source}

National Cancer Institute. IgA Nephropathy, Liver Disease-associated. NCI Thesaurus.

Code C121210.

IgA nephropathy secondary to hepatobiliary disease. 